\title{
Stimulation of the Lateral Habenula Inhibits Dopamine-Containing Neurons in the Substantia Nigra and Ventral Tegmental Area of the Rat
}

\author{
Greg R. Christoph, Robert J. Leonzio, and Karen S. Wilcox \\ Central Research and Development Department, E. I. du Pont de Nemours \& Company, Experimental Station, \\ Wilmington, Delaware 19898
}

\begin{abstract}
Neurons in the lateral habenula (LHb) of rats have efferent projections that terminate in the substantia nigra pars compacta (SNC) and ventral tegmental area (VTA), where cell bodies of dopamine-containing neurons are located. In order to study the influence of the habenula on dopaminergic activity, single-cell electrophysiological techniques were used to record unit discharge of dopamine-containing neurons in the SNC and VTA during electrical stimulation of the LHb or adjacent structures. Dopamine-containing neurons in the SNC and VTA were identified by their characteristic spike duration ( $>2 \mathrm{msec}$ ), discharge rate (2-8 spikes/sec), and irregular firing pattern. Analysis of peristimulus time histograms showed that $85 \%$ of $\mathrm{SNC}$ cells and $91 \%$ of VTA neurons were inhibited after single pulse stimulation ( $0.25 \mathrm{~mA}, 0.1 \mathrm{msec})$ of the LHb. The mean time between stimulation and onset of inhibition was $11 \mathrm{msec}$ (range, $2-22 \mathrm{msec}$ ) and mean duration of maximal suppression was 76 msec (range, 20-250 msec). Stimulation of structures adjacent to the LHb (hippocampus, lateral thalamus, medial dorsal thalamus, medial habenula) had little or no effect. Destruction of the fasciculus retroflexus, the fiber pathway that contains most habenular efferents, blocked the stimulation effects on dopamine-containing neurons. Destruction of the stria medullaris, which contains most habenular afferents, did not alter the inhibitory effect of habenular stimulation. Injection of a cytotoxin, kainic acid, in the $\mathrm{LHb} 1$ week before recording sessions blocked the inhibitory consequences of habenular stimulation. These experiments show that activation of ncuronal perikarya in the L.Hb causes orthodromic inhibition of dopamine-containing neurons in SNC and VTA via the fasciculus retroflexus.
\end{abstract}

The habenula of the rat is an epithalamic structure with afferents from cortical, limbic, and basal ganglia sources (Greatrex and Phillipson, 1982; Herkenham and Nauta, 1977). Efferents from the lateral habenula ( $\mathrm{LHb}$ ) project via the fasciculus retroflexus to numerous target structures, including the substantia nigra pars compacta (SNC) and the ventral tegmental area (VTA) (Herkenham and Nauta, 1979). The anatomical evidence that links the $\mathrm{LHb}$ with the SNC and VTA suggests that the LHb may exert an influence on dopamine-containing neurons whose perikarya are located in the SNC and VTA. With the exception of a report on the ability of lesions of the habenula to increase dopamine utilization in frontal cortex (Lisoprawski et al., 1980), there is no direct evidence that habenular efferents control the activity of dopamine-containing neurons. The present investi-

Received Dec. 17, 1984; revised June 10, 1985; accepted June 11, 1985.

We thank Beth A. Burkhart for technical assistance and Yronne J. King for preparation of the manuscript.

Correspondence should be addressed to Dr. Christoph.

Copyright (C) 1986 Society for Neuroscience 0270-6474/86/030613-07\$02.00/0 gation provides physiological evidence about the functional relationship between the LIIb and dopaminergic systems. This relationship was studied by electrical stimulation of the $\mathrm{LHb}$ during single-unit extracellular recording of dopamine-containing neurons in the SNC and VTA of anesthetized rats.

The stria medullaris is the major pathway of habenular afferents, and the fasciculus retroflexus contains all habenular efferents (Herkenham and Nauta, 1977, 1979). Electrolytic lesions of these fiber bundles permitted determination of which pathway was necessary for habenular stimulation to affect dopaminergic activity. Lesions of the $\mathrm{LHb}$ with kainic acid, in a concentration reported to selectively destroy ncuronal pcrikarya in the habenula (Contestabile and Villani, 1983), were made to specify whether the stimulation effects were conveyed by habenular efferents or by fibers of passage through the habenula (Herkenham and Nauta, 1977; Iwahori, 1977).

\section{Materials and Methods}

\section{Stimulation and recording procedures}

Male Sprague-Dawley rats, weighing 250-300 gm, were caged in groups and maintained on a $12 / 12 \mathrm{hr}$ light-dark cycle with food and water ad lib. Rats were anesthetized with chloral hydrate $(400 \mathrm{mg} / \mathrm{kg}$, i.p. $)$ and placed in a stereotaxic instrument. A concentric bipolar tungsten probe (Rhodes SNE-100) served as a stimulating electrode and was lowercd to the LHb at stereotaxic coordinates that correspond to atlas coordinates AP 3.6-4.0, ML 0.5-1.0, and DV 0.9-0.0 (Pellegrino et al., 1979).

Glass micropipettes used for recording extracellular single-unit activity were filled with $2 \mathrm{M} \mathrm{NaCl}$ that was saturated with Fast green or contained 2\% Pontamine sky blue and had tip diameters of 1-2 $\mu \mathrm{m}$ $(3.0-4.5 \mathrm{M} \Omega$ at $135 \mathrm{~Hz}$ ). The microelectrode was positioned dorsal to the recording site at a location corresponding to the following atlas coordinates: SNC, AP 1.9-2.2, ML 1.8-2.2, DV-2.0; VTA, AP 2.4-2.7, ML $0.4-0.8$, DV-1.8. The electrode was lowered with a hydraulic microdrive system and extracellular single-neuron action potentials were amplified, bandpass-filtered $(300-3000 \mathrm{~Hz})$, displayed visually, and monitored on audio with conventional equipment. Action potentials triggered a window discriminator whose output was integrated and displayed in ratemeter form. A laboratory computer (Mcdical Systcms Corp.) collected unit data and generated peristimulus time histograms.

Identification of dopamine-containing neurons was based primarily on electrophysiological characteristics (Grace and Bunney, 1983). Presumed dopamine-containing neurons had triphasic positive-negativepositive signals, and the duration of the positive-negative portion was 2-3 msec. The firing pattern was irregular, sometimes bursting, and the spontaneous firing rate was $2-7$ spikes/sec (mean, 4.6 spikes $/ \mathrm{sec}$ ). In other studies in our laboratory (unpublished observations), neurons with these electrophysiological characteristics had typical responses (Bunney et al., 1973) to systemic administration of dopaminergic agonists and antagonists. Neurons in the region of the SNC and VTA with biphasic action potential durations of $1.5 \mathrm{msec}$ or less and firing rates of 10-22 spikes/sec were presumed to be non-dopaminergic.

In all experiments, the spontaneous firing ratc of the ncuron main- 
Inhibit/excite

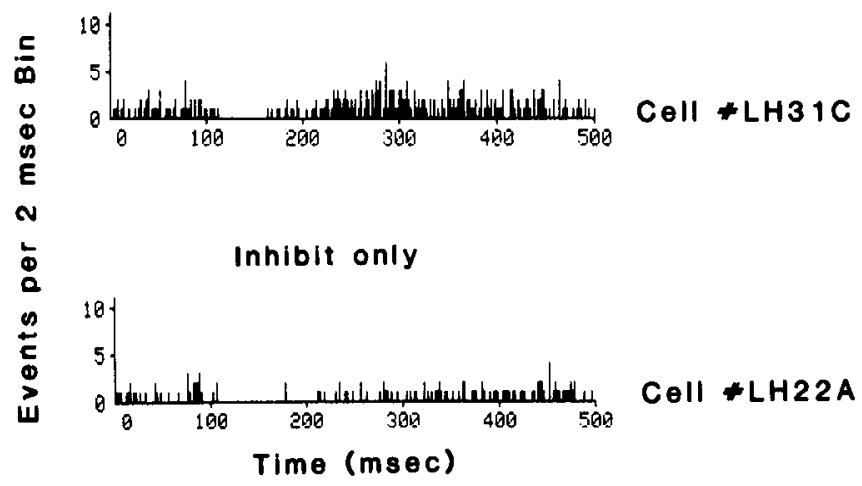

Figure 1. Typical responses of presumed dopamine-containing neurons to electrical stimulation of the lateral habenula. Stimulation pulses were delivered $100 \mathrm{msec}$ after initiation of each of 100 sweeps. Each sweep was $1500 \mathrm{msec}$ in duration, and only the first $500 \mathrm{msec}(250$ bins) of the histogram is shown.

tained a stable baseline for at least $3 \mathrm{~min}$ before stimulation of the $\mathrm{LHb}$ or adjacent structures. Constant-current stimulation pulses $(0.25 \mathrm{~mA}$, $0.1 \mathrm{msec}$ ) were delivered at a frequency of $0.67 \mathrm{~Hz}$. Dopamine-containing neurons were tested with variation of pulse current $(0.0-1.0 \mathrm{~mA})$ and pulse duration $(0.1-0.5 \mathrm{msec})$. Sites in the vicinity of the LHb, including the medial habenula, hippocampus, medial dorsal thalamus, and lateral thalamus, were stimulated in order to compare the effects to those of LHb stimulation. Stimulation of the LHb with 1 min trains of pulses $(5-20 \mathrm{~Hz})$ was used to measure the effects of repetitive stimulation on overall firing rate of dopaminergic neurons in the VTA. Although the majority of recording sites was ipsilateral to the stimulation site, a group of VTA neurons was tested with stimulation of the contralateral LHb.

In most experiments, four to eight neurons were tested in each rat. Dye spots marking the tip of the microelectrode were made at the end of the experiment by passing $-20 \mu \mathrm{A} \mathrm{DC}$ through the pipette for $15-$ $30 \mathrm{~min}$. The rats were perfused intracardially with $0.9 \%$ saline, followed by $10 \%$ formalin. The brains were removed and $40-\mu \mathrm{m}$-thick sections were mounted and stained with cresyl violet.

\section{Electrolytic lesions}

Lesions of the stria medullaris (four rats) and fasciculus retroflexus (four rats) were made by passing $0.5 \mathrm{~mA}$ anodal $\mathrm{DC}$ for $15-20 \mathrm{sec}$ through the core of the same type of electrode used for stimulation. Lesion sites were histologically confirmed as destroying the stria medullaris at AP 6.2, ML 0.5, and DV 0.2 and the fasciculus retroflexus at AP 3.3, ML $0.7, \mathrm{DV}-1.0$. In these rats, only SNC neurons were studied, and the time between lesioning and testing was at least $1 \mathrm{hr}$.

\section{Kainic acid lesions}

Kainic acid ( $1 \mathrm{ng} / \mathrm{nl}$ in $0.9 \%$ saline) was injected into the $\mathrm{LHb}$ from a micropipette (7-10 $\mu \mathrm{m}$ tip) with a hydraulically coupled pump (WP Instruments) at a rate of $25 \mathrm{nl} / \mathrm{min}$. The volume injected was either 125 $\mathrm{nl}$ (five rats) or $250 \mathrm{nl}$ (five rats). Two rats received $250 \mathrm{nl}$ of vehicle. The micropipette remained in position $5 \mathrm{~min}$ after the injection and was then slowly removed. Stimulation and recording sessions occurred 7-8 d after surgery. Dopamine-containing neurons in both SNC and VTA were sampled in each rat. In addition to cresyl violet staining, alternate sections from the kainate-injected rats were processed according to the silver stain procedure (method II) described by Fink and Heimor (1967).

\section{Results}

\section{Stimulation of $\mathrm{LHb}$ and adjacent structures}

Analysis of peristimulus time histograms of unit discharge of dopamine-containing neurons revealed that $85 \%$ of SNC neurons (44/52) and $91 \%$ of VTA neurons (50/55) were completely suppressed by electrical stimulation $(0.25 \mathrm{~mA}, 0.1 \mathrm{msec}$ pulse $)$ of the LHb. The mean duration of complete suppression for
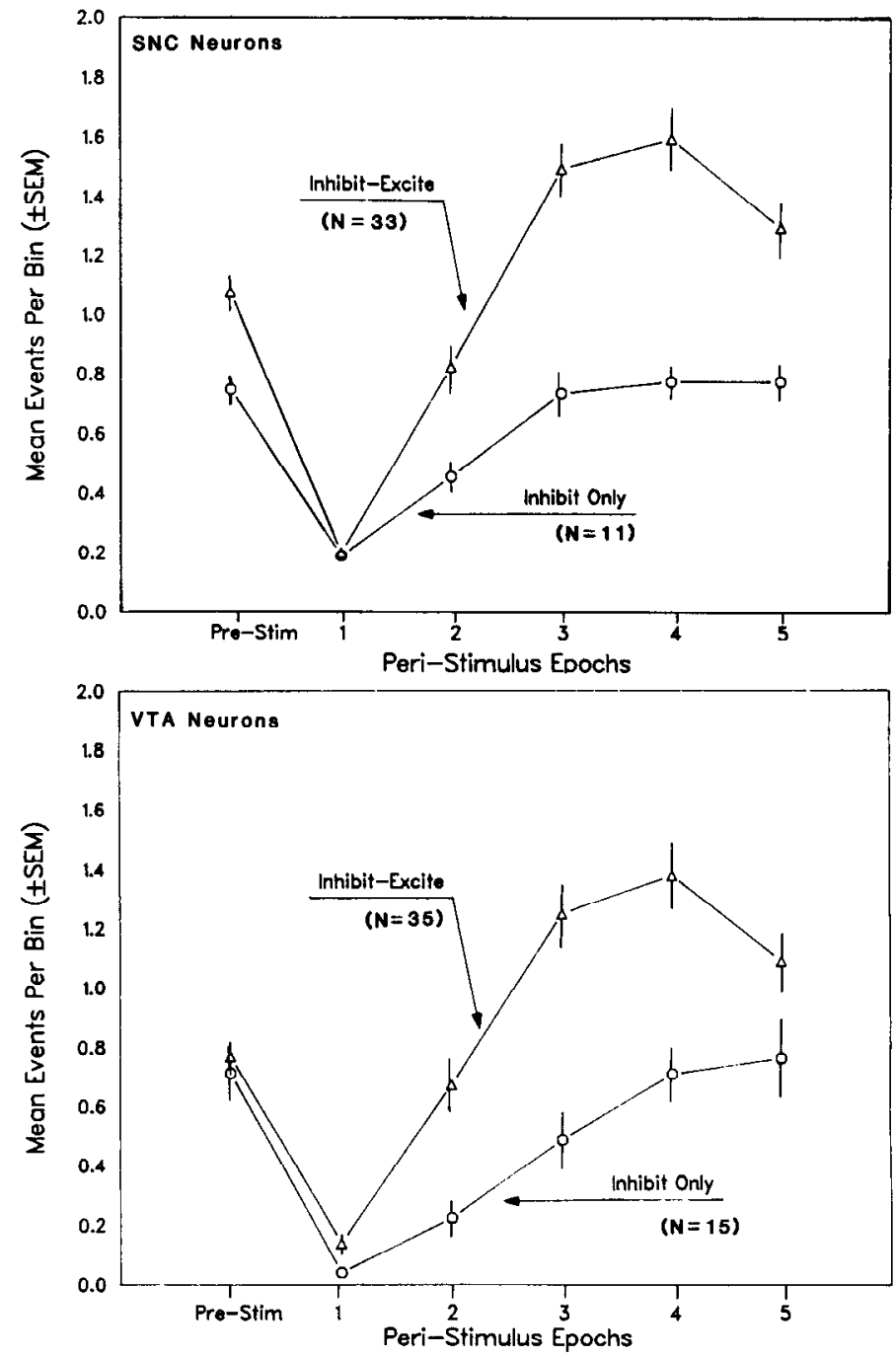

Figure 2. Peristimulus histograms of dopamine-containing neurons that responded to lateral habenula stimulation $(0.25 \mathrm{~mA}, 0.1 \mathrm{msec}, 0.67$ $\mathrm{Hz}$ ) were divided into epochs. Pre-Stim, 0-100 msec prior to the stimulation pulse; Epochs, 1-5, successive $50 \mathrm{msec}$ epochs beginning 20 mscc after the stimulation pulse, that is, 20-70, 70-120, 120-170, 170220 , and $220-270 \mathrm{msec}$ poststimulation. The spike events per bin per epoch were averaged for the two categories of response, Inhibit-Excite and Inhibit Only, that were detected in the substantia nigra pars compacta (SNC, top) and the ventral tegmental area (VTA, bottom). The response characteristics of SNC and VTA neurons were essentially the same.

these responsive neurons was $76 \mathrm{msec}$ (range, 20-250 $\mathrm{msec}$ ), and the mean time between stimulation and onset of suppression was $11 \mathrm{msec}$ (range, 2-22 msec).

For the purposes of categorization, a response was counted as inhibitory if the probability of spike events during a poststimulus epoch $50 \mathrm{msec}$ in duration was reduced by $50 \%$ or more with respect to a prestimulus epoch. A response was counted as excitatory if the probability of firing during a poststimulus epoch exceeded the control value by $30 \%$ or more. Two major categories of response were observed in both SNC and VTA. The largest category (64\%) showed inhibition followed by excitation, whereas most other responsive neurons $(24 \%)$ were only inhibited. Figure 1 shows typical histogram records for the two types of response, and Figure 2 displays the averaged data for successive temporal epochs of all histograms in a given subgroup. There were no statistically significant differences $\left(\chi^{2}\right.$ 
Cell +LH36A
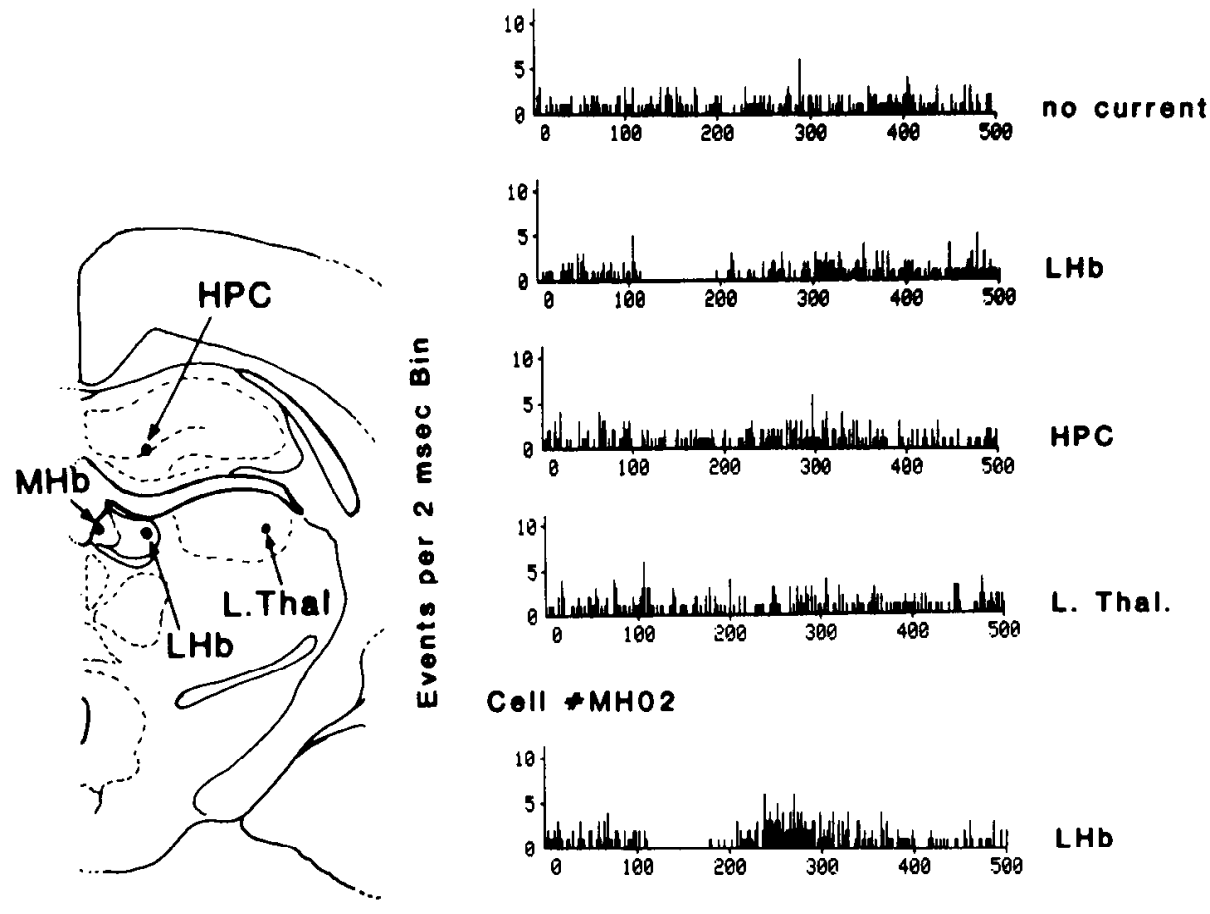

\section{Coll $+\mathrm{MHO} 2$}
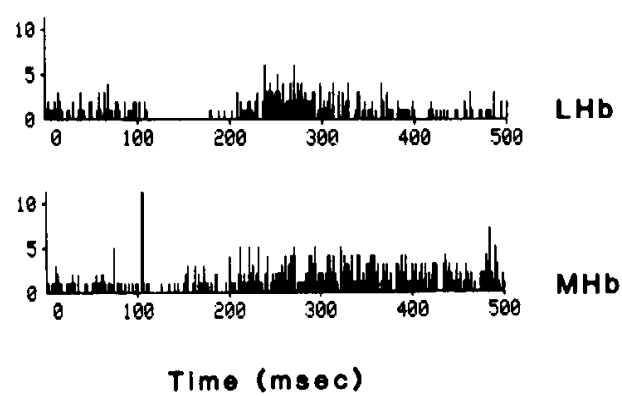

Figure 3. Stimulation of the lateral habenula inhibits dopamine-containing neurons in the VTA, whereas stimulation of adjacent sites does not. Schematic of brain section shows sites stimulated. Top four histograms, Results from the same neuron at different times with no stimulation current, stimulation of the lateral habenula $(L H b)$, hippocampus $(H P C)$, or lateral thalamus (L. Thal.). Bottom two histograms show a different VTA neuron, comparing the stimulation effects of the $\mathrm{LHb}$ and medial habenula $(M H b)$. All histograms consist of 100 sweeps, and the stimulus $(0.25 \mathrm{~mA}, 0.1 \mathrm{msec})$ was delivered $100 \mathrm{msec}$ after initiation of the sweep. tests) between SNC and VTA dopaminergic neurons with respect to the proportion of responsive cells or to the type of response (Table 1). In both SNC and VTA, the time between onset of inhibition and recovery to the prestimulus level of activity was significantly greater $(t$ tests, all $p<0.001)$ for the Inhibit Only response (SNC $=160 \mathrm{msec}, \mathrm{VTA}=180 \mathrm{msec}$ ) than for the Inhibit/Excite type $(\mathrm{SNC}=106 \mathrm{msec}, \mathrm{VTA}=112$ msec). For cells in the Inhibit/Excite subgroup, the mean time between stimulation and peak excitation was similar in SNC $(170 \mathrm{msec})$ and VTA (180 $\mathrm{msec})$.

Histological analysis showed that the tip of the stimulating electrode track was within the $\mathrm{LHb}$ in all rats for which $\mathrm{LHb}$ stimulation results are reported. Dye spots marking the location of dopamine-containing neurons were within the SNC or VTA. Electrode tracks on which the dye marking technique was not used were observed to penetrate the SNC and/or VTA.

Variation of stimulation pulse parameters with 15 dopaminecontaining neurons showed that increasing the pulse duration $(0.1-0.5 \mathrm{msec})$ or the stimulation current $(0.0-1.0 \mathrm{~mA})$ increased the duration of inhibition. With a fixed pulse duration $(0.1 \mathrm{msec})$, the minimal current required to elicit an inhibitory response was $0.125 \mathrm{~mA}$.

In nine rats, recording of a single neuron was maintaincd while varying the placement of the stimulating electrode in LHb, hippocampus, and lateral thalamus. Figure 3 shows a representative example in which LHb stimulation caused inhibition of a neuron in VTA, whereas stimulation of the other sites did not. Twelve VTA ncurons were tested with stimulation of the medial habenula $(0.25 \mathrm{~mA}, 0.1 \mathrm{msec}$ pulse). Two of these neurons were slightly inhibited with subsequent excitation, three cells showed only the excitatory component (Fig. 3), and seven others were unaffected. Stimulation of the medial dorsal thalamus at a site ventrolateral and anterior to the $\mathrm{LHb}$, which thereby avoided activation of the fasciculus retroflexus, had no effect on dopamine-containing neurons $(n=14)$. Fifteen of $17(88 \%)$ VTA neurons tested with stimulation of the contralateral LHb were inhibited.

Thirteen of 40 of the non-dopaminergic neurons in the SNC and VTA were inhibited by LHb stimulation, and when inhibition occurred, it was of shorter duration $(<30 \mathrm{msec})$ than that observed with dopamine-containing neurons. Nine non-dopaminergic neurons had a slight excitatory response and 18 were unresponsive.

\section{Effects of stimulus trains on firing rate}

The firing rate of dopamine-containing neurons was reduced during $1 \mathrm{~min}$ trains of pulses delivered to the $\mathrm{LHb}$. The amount of suppression was directly related to stimulation frequency (5-

Table 1. Effects of stimulation of the LHb on dopamine-containing neurons

\begin{tabular}{|c|c|c|c|}
\hline \multirow[b]{2}{*}{ Response } & \multicolumn{3}{|c|}{ Number of neurons ( $\%$ of column total) } \\
\hline & $\overline{\mathrm{SNC}}$ & VTA & Total \\
\hline Inhibit/excite & $33(63 \%)$ & $35(64 \%)$ & $68(64 \%)$ \\
\hline Inhibit only & $11(21 \%)$ & $15(27 \%)$ & $26(24 \%)$ \\
\hline Other response $e^{a}$ & $2(4 \%)$ & $1(2 \%)$ & $3(3 \%)$ \\
\hline No response & $6(12 \%)$ & $4(7 \%)$ & $10(9 \%)$ \\
\hline Total & $52(100 \%)$ & $55(100 \%)$ & $107(100 \%)$ \\
\hline
\end{tabular}

${ }^{a}$ Includes atypical response patterns such as excitation only or excitation imbedded within a longer inhibitory period. 


\section{Cell *LHb-stim 011}

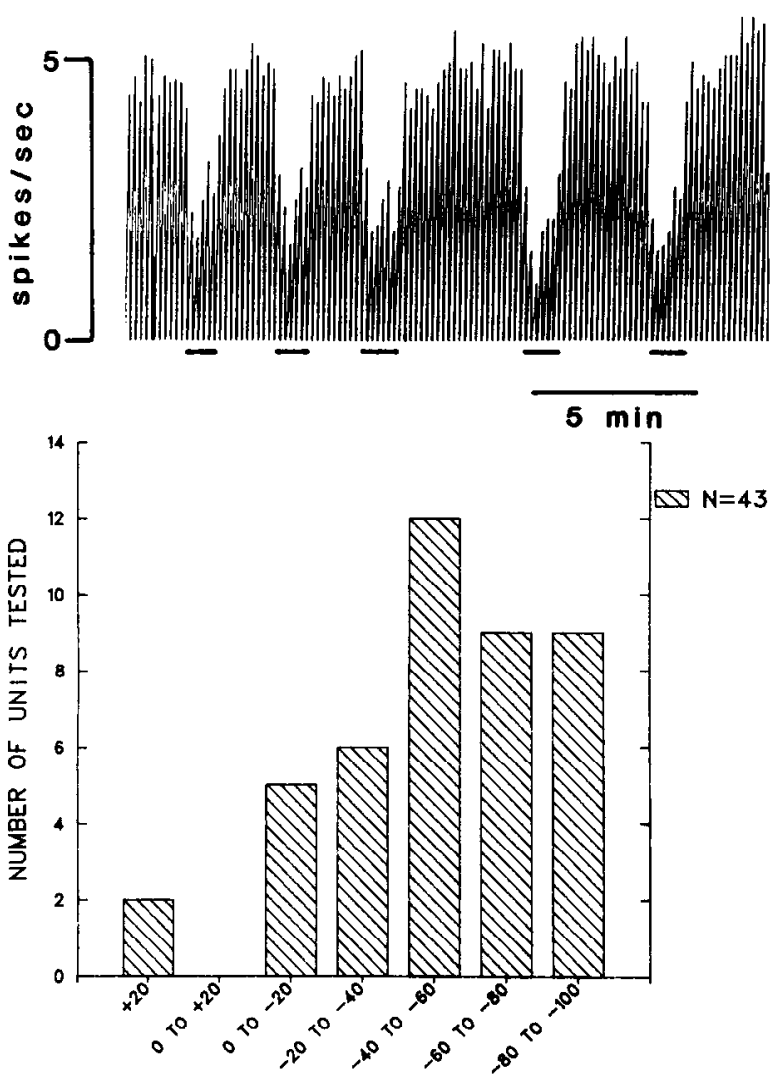

PERCENT CHANGE IN FIRING RATE

Figure 4. Top, Ratemeter record of dopamine-containing neuron in the VTA. Stimulation of the $\mathrm{LHb}(10 \mathrm{~Hz}, 0.25 \mathrm{~mA}, 0.1 \mathrm{msec})$ at the times indicated suppressed the overall firing rate of the cell. Bottom, Histogram showing the number of VTA dopaminergic cells that responded to habenular stimulation $(10 \mathrm{~Hz}, 0.25 \mathrm{~mA}, 0.1 \mathrm{msec})$. The response categories on the abscissa represent the percentage change in firing rate during the $1 \mathrm{~min}$ stimulation period.

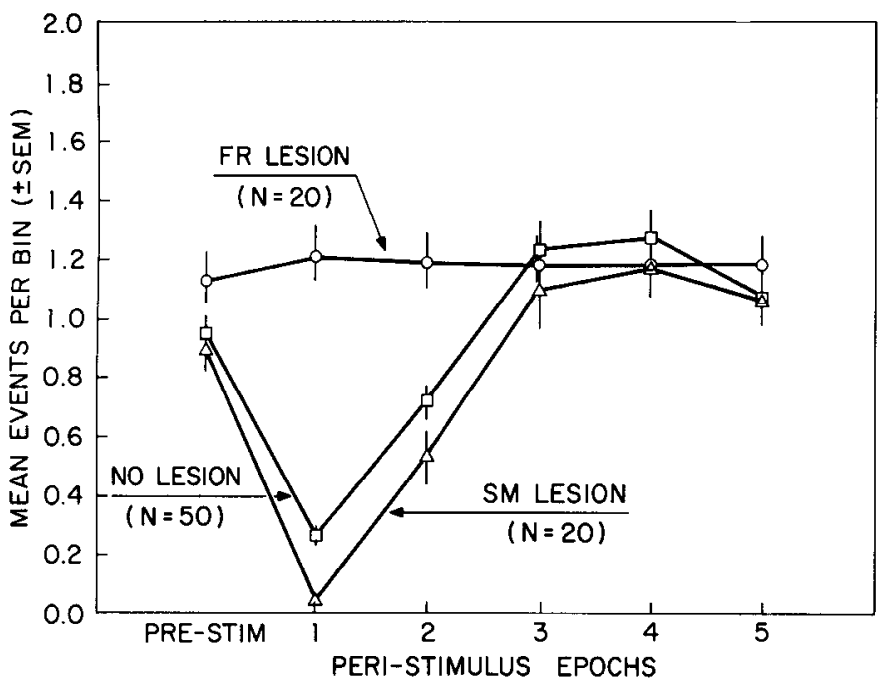

Figure 5. Effects of lesions of the principal habenular afferent and efferent fiber bundles on the responsiveness of dopamine-containing SNC neurons to stimulation of the LHb. Peristimulus time histogram epochs are as defined in Figure 2. The mean events per bin per epoch are shown for three groups of rats. Stimulation of the No Lesion control group suppressed neuronal activity during poststimulus epochs 1 and
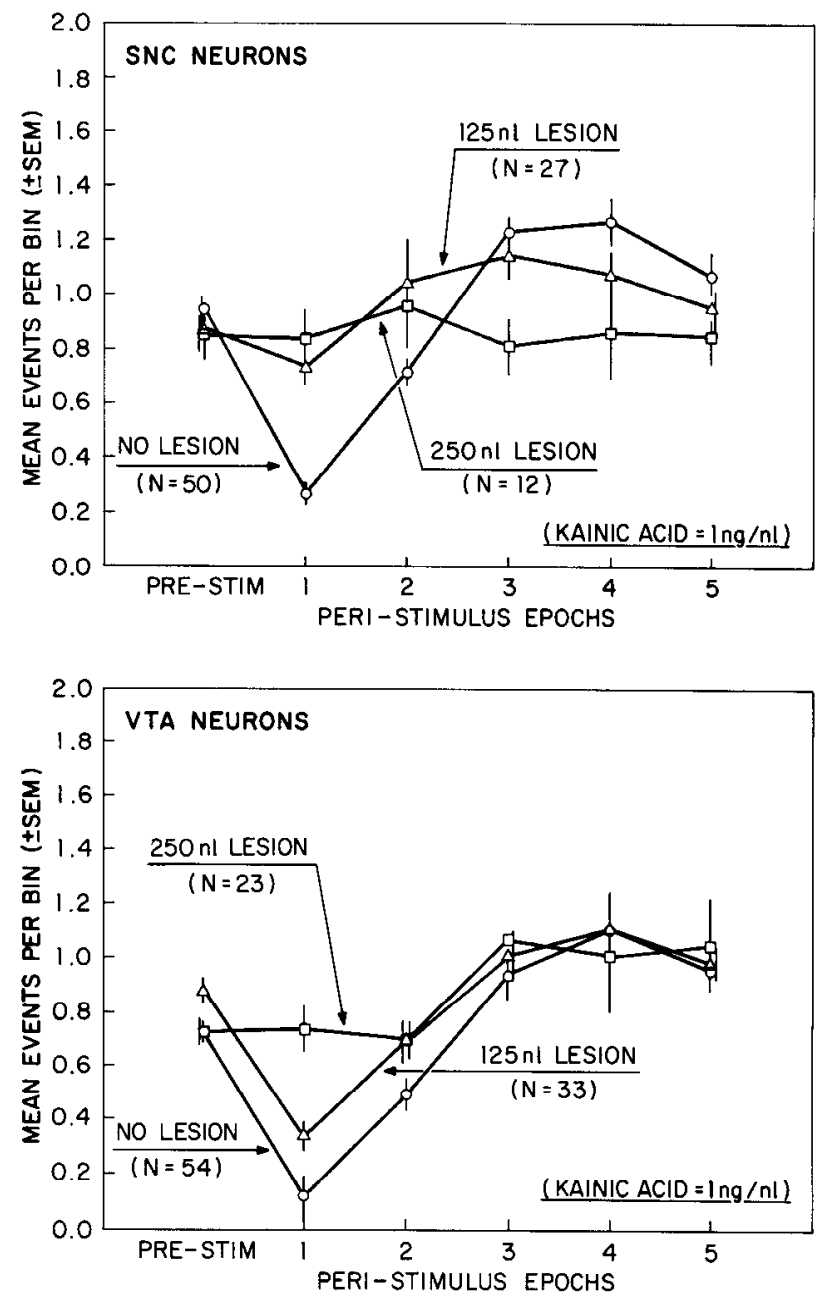

Figure 6. Effects of kainic acid lesions of the $\mathrm{LHb}$ on the responsiveness of dopaminergic ncurons to stimulation of the LHb. Mean spike events per bin per epoch were averaged over all cells in a given group and are displayed as a function of peristimulus epochs defined as in Figure 2. Top, Inhibitory effects of habenular stimulation $(0.25 \mathrm{~mA}, 0.1$ msec, $0.67 \mathrm{~Hz}$ ) on neurons in the $S N C$ were blocked by prior injection of either 125 or $250 \mathrm{nl}$ kainic acid into the LHb. Bottom, Dopaminecontaining neurons in the $V T A$ were inhibited by habenular stimulation in the No Lesion group and the $125 \mathrm{nl}$ Lesion group but were not inhibited in the $250 \mathrm{nl}$ Lesion group.

$20 \mathrm{~Hz}, n=8$ ). All of a group of VTA dopaminergic neurons $(n=43)$ were tested with identical stimulation parameters $(0.25$ $\mathrm{mA}, 0.1 \mathrm{msec}$ pulse, $10 \mathrm{~Hz}$ ). The firing rate during the stimulation period was expressed as a percentage of the firing rate immediately preceding the stimulation period. Figure 3 shows that firing rate was slowed by more than $20 \%$ in 36 of the 43 neurons tested. Two cells were excited by more than $20 \%$, and five cells were not clearly affected. The modal response was approximately $50 \%$ reduction in firing rate.

\section{Antidromic activation}

Throughout the study, neurons were examined for evidence of antidromic activation caused by habenular stimulation. Of 57 SNC neurons and 83 VTA neurons tested in nonlesioned rats, evidence of antidromic activation was detected in only three

\section{$\leftarrow$}

2. Electrolytic lesion of the stria medullaris (SM Lesion) did not substantially alter the response, whereas the response was completely blocked after lesion of the fasciculus retroflexus (FR Lesion). 


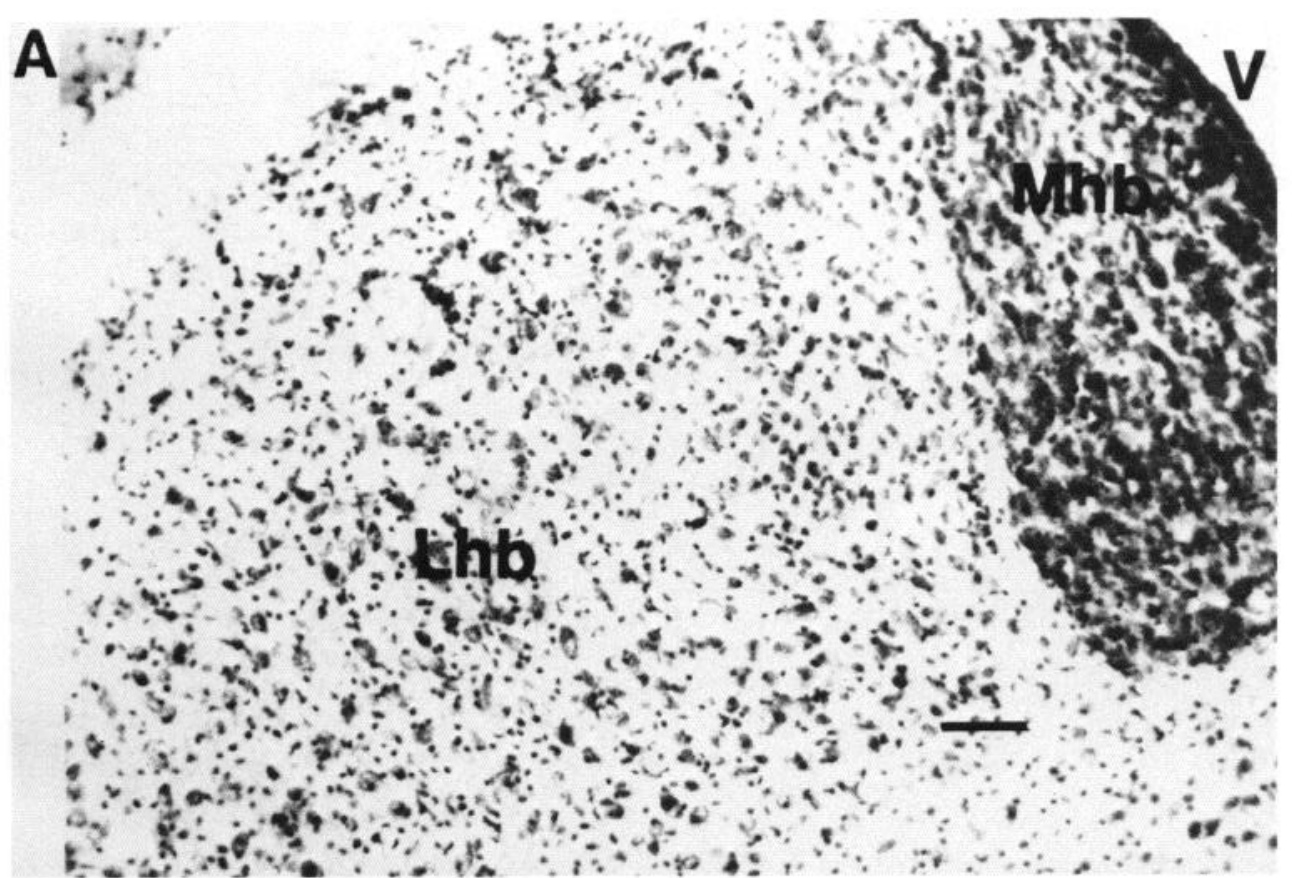

B

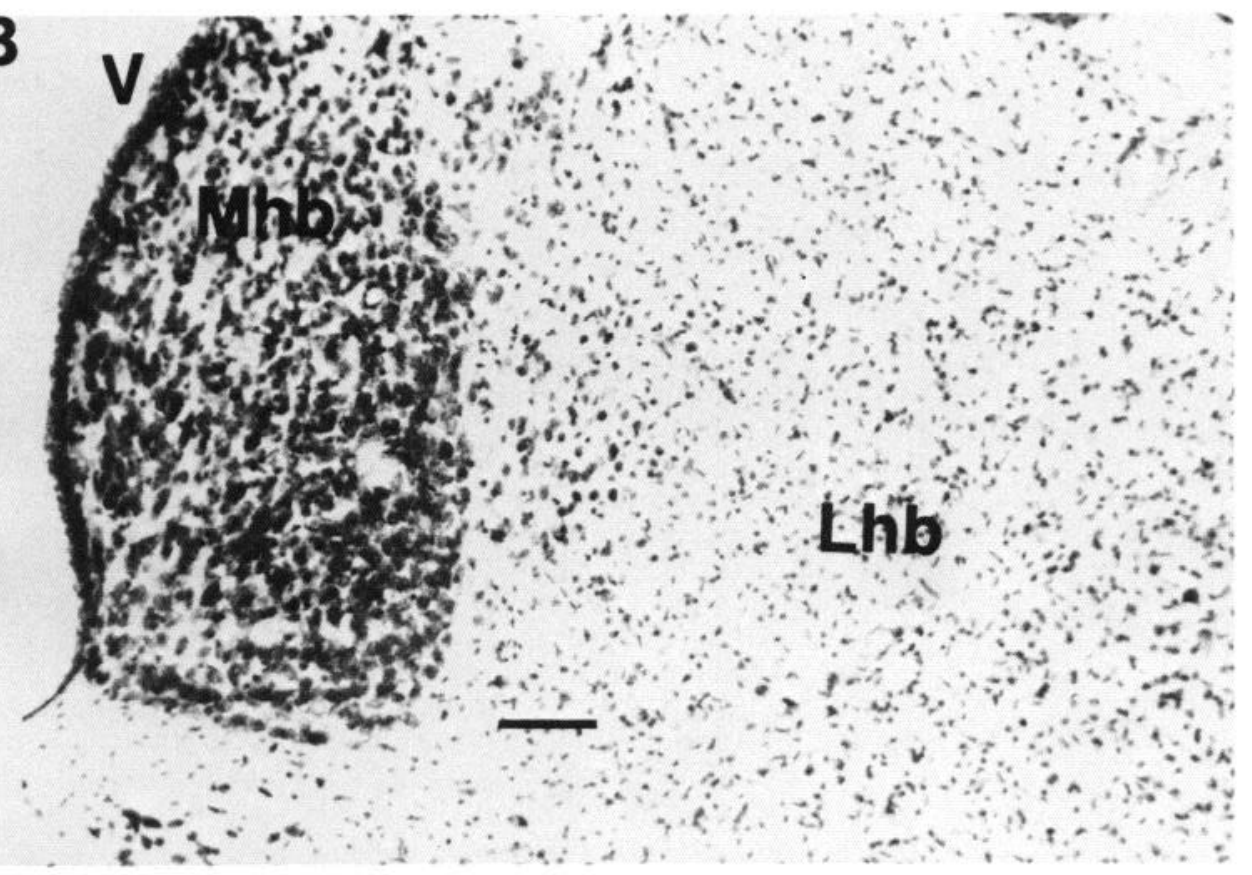

Figure 7. Photomicrographs of $(A)$ an untreated habenula and $(B)$ the contralateral kainic acid-treated $(250 \mathrm{nl})$ habenula. Both have been stained with cresyl violet. Note the lack of neuronal cell bodies and the proliferation of glial cells in the $L H b$ in $B$. The $M H b$ has not been damaged. Bar, $100 \mu \mathrm{m}$. $L H b$, Lateral habenula; $M H b$, medial habenula; $V$, lateral ventricle. cells in the VTA. Latency of the antidromic spike $(2.5-3.0 \mathrm{msec})$ was highly reproducible. Only one of these neurons was recorded for a long enough period to confirm the antidromic nature of the spike with tests of collision and ability to follow double pulses separated by $5 \mathrm{msec}$ (data not shown).

\section{Fasciculus retroflexus and stria medullaris lesions}

Peristimulus time histograms of SNC cells in rats with fasciculus retroflexus lesions or stria medullaris lesions were compared to those of SNC cells in rats without lesions. Figure 5 shows that the fasciculus retroflexus lesions blocked the effect of LHb stimulation in 19 of 20 neurons. In rats with stria medullaris lesions, all 20 cells tested were inhibited, and in the untreated control group, 44 of 52 neurons were inhibited.
Kainic acid lesions of the $\mathrm{LHb}$

The inhibitory effect of habenular stimulation was completely blocked by the $250 \mathrm{nl}$ injection of kainic acid. None of the dopamine-containing neurons in SNC $(n=12)$ or VTA $(n=$ 23) were inhibited by more than $30 \%$ in the $250 \mathrm{nl}$ group. The $125 \mathrm{nl}$ injection also blocked the inhibitory effect of habenular stimulation on most SNC neurons; only four of 27 neurons were inhibited. VTA neurons, however, were as frequently inhibited in the $125 \mathrm{nl}$ group as in the untreated controls (29/33 and 50/ 55 cells inhibited, respectively). Figure 6 shows the blocking effect of kainic acid on the inhibitory response; it also shows that excitatory responses occurred 150-200 msec after stimulation even in the absence of a prior inhibition. This was par- 

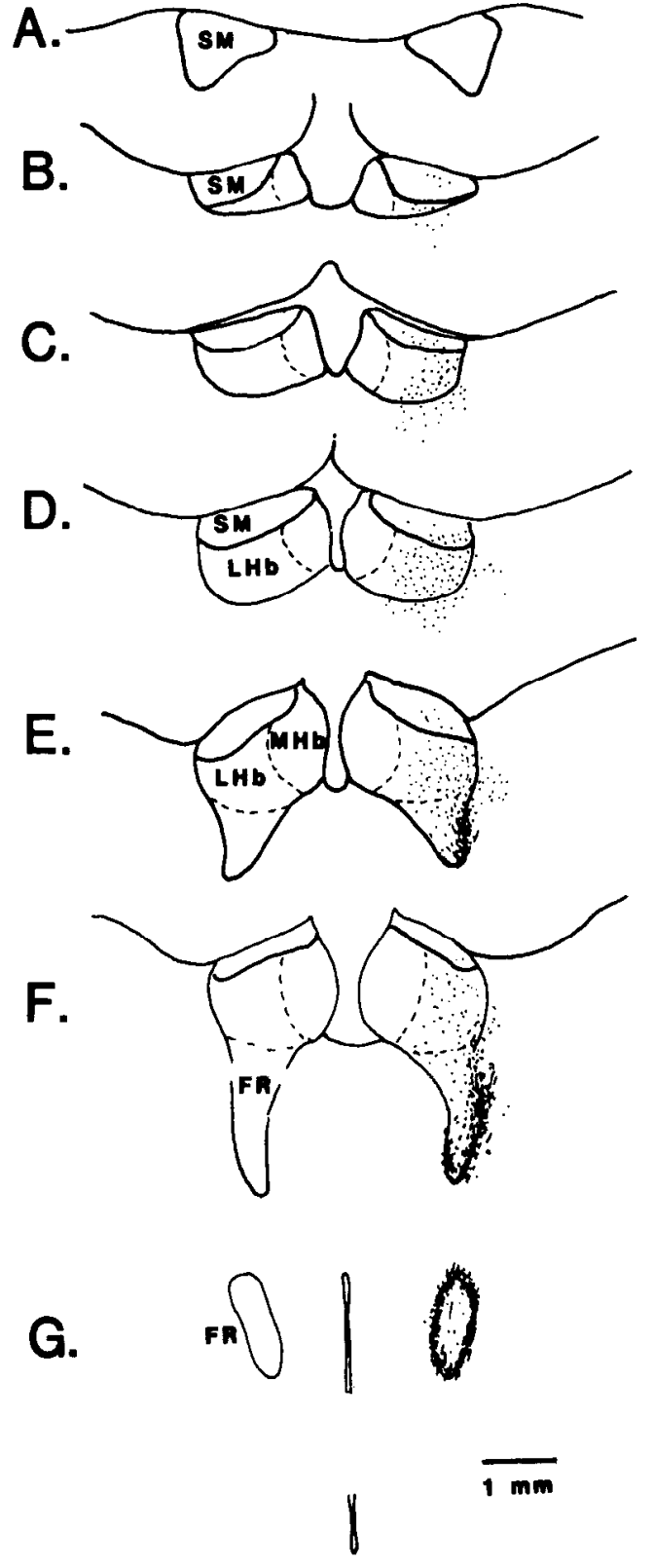

H.
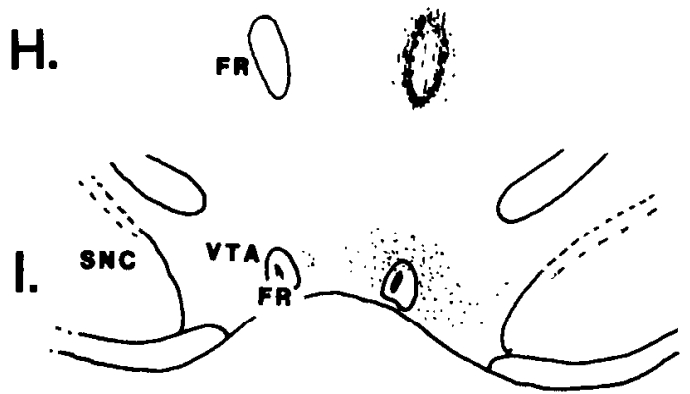

Figure 8. Neuronal degeneration 1 week after injection of $250 \mathrm{nl}$ kainic acid $(1 \mathrm{ng} / \mathrm{nl})$ into the $L H b$ of a rat. $A-I$, Line drawings of relevant structures and dot representation of the areas of degeneration. Panel $A$ is most anterior and $I$ most posterior. The injection site was between panels $D$ and $E$. Note the absence of degeneration in the stria medularis ( $S M$ in $A$ ) and the dense staining surrounding the core of the fasciculus retroflexus ( $F R$ in $F-H)$. No kainate-induced degeneration occurred in the medial habenula $(M H b)$ nor in any area contralateral to the side of injection. ticularly evident in the VTA with rats that received $250 \mathrm{nl}$ of kainic acid. Fourteen of $23(61 \%)$ neurons in this group showed the excitatory response. Stimulation of the LHb in the two rats that were injected with vehicle instead of kainic acid caused inhibition in 13 of 15 dopaminergic neurons tested (data not shown).

The brains of the rats that received kainic acid or vehicle injections were sectioned and treated with a silver stain in order to visualize tissue degeneration. The area surrounding the injection pipette was characterized by a zone of argyrophilic material presumed to be cellular debris (Fink and Heimer, 1967). This zone corresponded to elimination of neuronal perikarya, as demonstrated by cresyl violet histology (Fig. 7), and was confined to the lateral aspect of the LHb with the $125 \mathrm{nl}$ injection. With the $250 \mathrm{nl}$ group, the damaged zone extended into the medial aspect of the LHb but did not include the medial habenula. For the rats in the $250 \mathrm{nl}$ group, the damaged area included approximately $200-500 \mu \mathrm{m}$ of adjacent thalamus ventral and lateral to the $\mathrm{LHb}$. Argyrophilic material characteristic of fiber degeneration was densest surrounding the core of the ipsilateral fasciculus retroflexus. The contralateral fasciculus retroflexus and contralateral $\mathrm{LHb}$ did not contain detectable degeneration. No retrograde degeneration was detected in the stria medullaris on either side of the brain at points more than 400 $500 \mu \mathrm{m}$ anterior to the LHb. Figure 8 shows a representation of the degeneration pattern for one of the rats in the $250 \mathrm{nl}$ group. No degeneration was detected in either of the two rats that received vehicle instead of kainic acid.

\section{Discussion}

The experimental results reported here show that electrical stimulation of the LHb causes inhibition of $85-91 \%$ of SNC and VTA neurons that have electrophysiological characteristics of dopamine-containing neurons. Stimulation of habenular neurons at $10 \mathrm{~Hz}$ caused a reduction in the firing rate of $84 \%$ of VTA dopaminergic neurons tested. The LHb has direct bilateral efferent projections to the SNC and VTA (Herkenham and Nauta, 1979), and stimulation of either the ipsilateral or contralateral LHb was equally effective in inhibiting DA-containing neurons. There is some evidence (Cuello et al., 1978; McGeer et al., 1977) that a subset of efferents from the medial habenula terminate in the VTA. Stimulation of the medial habenula, however, typically had little, if any, inhibitory effect on dopaminergic neurons.

There were no major differences between dopamine-containing neurons in SNC and VTA with respect to the effects of $\mathrm{LHb}$ stimulation. The reason that some SNC and VTA neurons displayed inhibition followed by excitation while others were only inhibited is unknown. Both types of response were often found on the same electrode track with no change in stimulation parameters. The excitatory component is probably not a rebound excitation because it was sometimes detected in the absence of a prior inhibition, particularly in kainate-treated rats and for a few cells tested with stimulation of the medial habenula.

Lesions of the stria medullaris that preceded recording SNC neurons provided data indicating that this afferent pathway is not required for habenular activation to inhibit dopamine-containing neurons. In contrast, lesions of the fasciculus retroflexus caused virtually complete blockade of the ability of habenular stimulation to inhibit SNC dopamine-containing neurons. Although this outcome demonstrates that an intact fasciculus retroflexus is necessary for habenular activation to suppress dopaminergic activity, it does not prove that $\mathrm{LHb}$ efferents within the fiber bundle mediate the neuronal response. Two alternate explanations are possible. First, activation of fibers that are known to pass through the habenula (Herkenham and Nauta, 
1979; Iwahori, 1977) could affect dopaminergic neuronal activity. Second, the fasciculus retroflexus contains some habenular afferents, a subset of which originates in the VTA (Skagerberg et al., 1984; Swanson, 1982). Indeed, antidromic activation of a few VTA neurons was detected in our work, and it is possible that antidromic activation of these habenular afferents, in conjunction with collateral inhibition, could cause widespread inhibition of dopamine-containing neurons. If either of these two alternate explanations were correct, then selective destruction of neuronal perikarya while sparing terminals and fibers of passage in the $\mathrm{LHb}$ would not block the response of dopaminergic neurons to $\mathrm{LHb}$ stimulation. The experiments with kainic acid lesions of the $\mathrm{LHb}$ were performed for this reason.

The results of the silver stain histology suggest that the kainic acid lesion in our study did not destroy terminals or fibers of passage. If terminals and fibers of passage had been damaged, retrograde degeneration would have been detected in the stria medullaris-as, for example, was detected in our laboratory in rats with electrolytic lesions of the LHb (unpublished observations). Since afferents and terminals entering the $\mathrm{LHb}$ via the stria medullaris were spared from damage, it is likely that efferents entering the $\mathrm{LHb}$ via the fasciculus retroflexus were also spared. Therefore, the degeneration surrounding the core of the fasciculus retroflexus probably represents anterograde degeneration that occurred as a result of destruction of neuronal perikarya in the LHb. The core of the fasciculus retroflexus principally contains efferents of the medial habenula, whereas $\mathrm{LHb}$ efferents are reported to surround the core of the fasciculus retroflexus (Herkenham and Nauta, 1979). Since lateral habenula and not medial habenula neurons were damaged by kainic acid, and we only saw degeneration surrounding the core of the fasciculus retroflexus, our work confirms these earlier neuroanatomical data.

Since kainate-induced destruction of neuronal perikarya in the LHb blocked the inhibitory effects of LIIb stimulation, it is unlikely that our results can be explained by antidromic activation and collateral inhibition or by activation of fibers of passage. Instead, the data indicate that stimulation of neuronal perikarya in the LHb causes orthodromic inhibition of dopaminergic neurons via the fasciculus retroflexus. The reason that a larger volume ( $250 \mathrm{vs} 125 \mathrm{ml}$ ) of kainic acid was required to block the inhibitory stimulation effects on VTA neurons may be related to the additional damage in the medial aspect of the $\mathrm{LHb}$ with the larger volume. Perhaps there is a topographical arrangement whereby medial $\mathrm{LHb}$ ncurons project to the VTA and lateral $\mathrm{LHb}$ neurons project to SNC.

Although the present evidence indicates that activation of LHb efferents suppresses the electrical activity of most dopamine-containing neurons, whether this effect truly represents dircct inhibition by monosynaptic inputs is not known. The conduction velocity of LHb efferents as determined by antidromic activation studies has been reported to vary between 0.4 and $8.5 \mathrm{~m} / \mathrm{sec}$ (Garland and Mogenson, 1983). If we estimate the length of the efferent pathway to be $4 \mathrm{~mm}$, the corresponding transit time to the SNC/VTA is between 10 and 0.4 $\mathrm{m} / \mathrm{sec}$. The mean onset time of inhibition was $11 \mathrm{msec}$ in our work, so although it is possible that dopamine-containing neurons are monosynaptically inhibited, a more complex arrangement involving one or more interneurons is plausible.

The anatomical connections of the habenula may confer on it the ability to integrate limbic, basal ganglia, and cortical activity for transmission to midbrain structures. For example, physiological studies (Stern et al., 1979; Wang and Aghajanian, 1977) and neurochemical measurements (Speciale et al., 1980) suggest that the habenula is a major link between forebrain structures and midbrain serotonin-containing neurons. The present study suggests that the habenula also serves as a link between forebrain structures and midbrain dopaminergic neurons.

\section{References}

Bunney, B. S., J. R. Walters, R. H. Roth, and G. K. Aghajanian (1973) Dopaminergic neurons: Effects of antipsychotic drugs and amphetamine on single cell activity. J. Pharmacol. Exp. Ther. 185:560-571.

Contestabile, A., and L. Villani (1983) The use of kainic acid for tracing neuroanatomical connections in the septohabenulointerpeduncular system of the rat. J. Comp. Neurol. 214: 459-469.

Cuello, A. C., P. C. Emson, G. Paxino, and T. Jessel (1978) Substance $P$ containing and cholinergic projections from the habenula. Brain Res. 149: 413-429.

Fink, R. P., and L. Heimer (1967) Two methods for selective silver impregnation of degenerating axons and their synaptic endings in the central nervous system. Brain Res. 4: 369-374.

Garland, J. C., and G. J. Mogenson (1983) An electrophysiological study of convergence of entopeduncular and lateral preoptic inputs on lateral habenular neurons projecting to the midbrain. Brain Res. 263: 33-41.

Grace, A. A., and B. S. Bunney (1983) Intracellular and extracellular electrophysiology of nigral dopaminergic neurons. I. Identification and characterization. J. Neurosci. 10: 301-315.

Greatrex, R. M., and O. T. Phillipson (1982) Demonstration of synaptic input from prefrontal cortex to the habenula in the rat. Brain Res. 238: 192-197.

Herkenham, M., and W. J. Nauta (1977) Afferent connections of the habenular nuclei in the rat. A horseradish peroxidase study with a note on the fiber-of-passage problem. J. Comp. Neurol. 173: 123146.

Herkenham, M., and W. Nauta (1979) Efferent connections of the habenular nuclei in the rat. J. Comp. Neurol. 187: 19-48.

Iwahori, N. (1977) A golgi study on the habenular nucleus of the cat. J. Comp. Neurol. 171: 319-344.

Lisoprawski, A., D. Herve, G. Blanc, J. Glowinski, and J. P. Tassin (1980) Selective activation of the mesocortico-frontal dopaminergic neurons induced by lesion of the habenula in the rat. Brain Res. 183: 229-234.

McGeer, P. L., E. G. McGeer, and T. Hattori (1977) Dopamineacetylcholine-GABA neuronal linkages in the extrapyramidal and limbic systems. Adv. Biochem. Psychopharmacol. 16: 397-402.

Pellegrino, L. J., A. S. Pellegrino, and A. J. Cushman (1979) A Stereotaxic Atlas of the Rat Brain, Plenum, New York.

Skagerberg, G., O. Lindvall, and A. Björklund (1984) Origin, course and termination of the mesohabenular dopamine pathway in the rat. Brain Res. 307: 99-108.

Speciale, S. G., L. M. Neckers, and R. J. Wyatt (1980) Habenular modulation of raphe indoleamine metabolism. Life Sci. 27: 23672372.

Stern, W., A. Johnson, J. D. Bronzino, and P. J. Morgane (1979) Effects of electrical stimulation of the lateral habenula on single-unit activity of raphe neurons. Exp. Neurol. 65: 326-343.

Swanson, L. W. (1982) The projection of the ventral tegmental area and adjacent regions: A combined fluorescent retrograde tracer and immunofluorescence study in the rat. Brain Res. Bull. 9: 321-353.

Wang, R. Y., and G. K. Aghajanian (1977) Physiological evidence for habenula as a major link between forebrain and midbrain raphe. Science 197: 89-91. 